\title{
Un matricide, figure des liens familiaux violents
}

\section{François Marty ${ }^{1}$}

\author{
Résumé \\ Si les meurtres familiaux ne sont pas les actes les plus fréquents dans \\ le registre de la violence au sein de la famille, et heureusement, ils n'en \\ constituent pas moins des exemples paradigmatiques de la violence à \\ l'adolescence, illustrant aussi bien la violence de l'adolescent que celle \\ de l'adolescence. Ces meurtres ne sont pas la réalisation de fantasmes, \\ mais plutôt le meurtre d'un fantasme œdipien. Ils traduisent un \\ fonctionnement narcissique, celui de l'adolescent mais aussi celui de sa \\ famille.
}

Mots clés

Parricide, Violence, Inceste, Fantasme de meurtre, Subjectivation

Si les meurtres familiaux ne sont pas les actes les plus fréquents dans le registre de la violence au sein de la famille, et heureusement ils n'en constituent pas moins des exemples paradigmatiques de la violence à l'adolescence, illustrant aussi bien la violence de l'adolescent que celle de l'adolescence. Ces meurtres ne sont pas la réalisation de fantasmes, mais plutôt le meurtre d'un fantasme œdipien. Ils traduisent un fonctionnement narcissique, celui de l'adolescent mais aussi celui de sa famille.

Partant du cas d'un adolescent âgé d'une vingtaine d'années au moment où il a commis

\footnotetext{
1 Psychologue, psychanalyste et professeur des universités. Il a été directeur de l'Institut de Psychologie (Université Paris Descartes) et du Laboratoire de Psychologie Clinique et Psychopathologie (LPCP) de 2007 à 2012. Il a été directeur du Collège International de l'Adolescence (CIA) de 2004 à 2012. Psychanalyste en liberal et directeur de la collection «Champs Libres» aux Éditions In Press (Paris). E-mail:fmarty2004@yahoo.fr
}

(c) Auteur 2021. Publié en ligne sur https://rppsicanalise.org, sous la licence Creative Commons Attribution-NonCommercial 4.O. ses crimes, je souhaite discuter l'idée selon laquelle l'acte meurtrier intra familial est très souvent d'abord l'expression d'une difficulté à se séparer - physiquement, mais surtout psychiquement - des autres membres de sa famille au moment de l'adolescence. Cette difficulté confine à l'impasse quant à la possibilité de se subjectiver. Ce crime de sang est aussi le signe d'une impossibilité à élaborer psychiquement les transformations pubertaires, sur fond d'impuissance à se venir en aide soi-même. Le rôle de l'environnement est prévalent dans ce type de crime, dans la mesure où le fonctionnement psychique familial est très souvent organisé sur le mode de l'indifférenciation, de l'incestualité. En somme, là où devrait advenir la génitalité au moment de l'apparition de la puberté pour l'adolescent, demeure l'organisation narcissique infantile prévalant dans la famille. Ce meurtre de l'autre familial apparaît comme une impasse à être, mais aussi comme l'exacte 
réplique d'une tentative pour changer de vie et renaître à la manière, paradoxale, de certaines formes de suicide.

La violence de l'acte dévoile la profondeur de la détresse à être pour ces adolescents et adolescentes qui ne trouvent d'autres issues que celle du passage à l'acte meurtrier, comme si les liens aux objets parentaux ne pouvaient se défaire qu'en tranchant dans le vif, comme si la liaison était désubjectivante et la déliaison dangereusement porteuse du risque de la folie. D'un côté, le lien asservit dans l'impossible individuation, de l'autre, la tentative de s'en défaire apparaît comme une perte des assises, un désarrimage au socle qui soutient le sujet. Cette situation n'est pas sans rappeler la folie incestuelle décrite par Racamier (1980, 1989) et les alliances inconscientes dont les pactes dénégatifs constituent une version destructrice du lien pour Kaës (2009). Dans tous les cas, il semble bien que soit entravée la subjectivation (Cahn, 1991), processus le plus dynamique au moment de l'adolescence. La violence du crime de sang est à l'image de la violence de l'enfermement que vit l'adolescent dans un espace familial vécu comme aliénant.

\section{L'HISTOIRE DE PIERRE RIVIÈRE}

C'est en cherchant dans les Archives du Calvados quelques documents relatifs à des affaires judiciaires au début du XIX ème siècle illustrant la façon dont la psychiatrie s'était introduite dans les prétoires que Jean Pierre Peter, historien et collaborateur de M. Foucault, et exhumé le dossier complet du procès de Pierre Rivière (Foucault et al., 1973), un jeune matricide et fratricide condamné à mort puis gracié par le Roi dans les années 1830-1835. Le cas de ce jeune adolescent est l'un de ceux qui permettra l'élaboration de la loi de 1838 sur la responsabilité pénale de certains criminels. La psychiatrie, discipline alors encore toute récente, affirmait par ailleurs que, dans certains cas, ces crimes étaient le fait de fous qui ne pouvaient être condamnés aux mêmes peines que ceux qui avaient toute leur raison au moment des faits qui leur étaient reprochés.
Le dossier judiciaire du cas Rivière nous apprend que l'auteur de ces crimes avait d'abord voulu expliquer au juge qu'il avait agi pour obéir à Dieu. Puis, dans une deuxième version, il donnait un autre récit qui mettait en évidence le problème conjugal que vivaient ses parents. C'est ce qui, disait-il, l'avait poussé à tuer sa mère pour protéger son père qui menaçait de se suicider face aux violentes attaques dont il était victime de la part de sa femme. La lecture du dossier nous apprend également que ce n'est pas seulement sa mère que Pierre Rivière a tuée mais aussi sa sœur cadette et son plus jeune frère. Lors de cette deuxième explication, moins divine et plus familiale, Pierre aurait parlé devant le juge pendant plus de deux heures de façon si fascinante que le greffier en aurait oublié de prendre la moindre note. C'est pour pallier à cet oubli que le juge demande à Pierre Rivière de mettre par écrit tout ce qu'il vient si bien de dire, lui ce petit paysan normand sans grande éducation qui pourtant cite volontiers les écritures saintes et fait référence à l'histoire de France. C'est ainsi que Pierre Rivière écrit cette déposition en prison, ce document un Mémoire - devenant pièce à conviction dans le procès.

Nous considérons ce Mémoire (Marty, 1989) comme un document clinique d'une richesse exceptionnelle dans la mesure où il retrace avec précision le cheminement mental de Pierre Rivière ou du moins la tentative désespérée qu'il fait pour expliquer son crime au juge. Davantage qu'un récit autobiographique, ce document nous aide à penser avec l'auteur de ces crimes ce qui — de son point de vue - a contribué à le pousser à ce passage à l'acte meurtrier. Il s'agit donc d'un témoignage en première personne sur une tentative de réflexion qui nous donne à penser sur le fonctionnement psychique de Pierre Rivière. Cette explication que l'auteur se donne à lui-même en même temps qu'il cherche à se faire comprendre par le juge a d'autant plus de valeur que c'est l'auteur lui-même qui nous la fournit, c'est lui qui choisit le chemin pour s'expliquer, les arguments pour convaincre. Cet effort pour se faire comprendre, comme il l'écrit lui-même, nous montre à quel point l'acte résiste à être 
expliqué, voire à être explicable. Ce n'est donc pas cette explication que nous retiendrons dans notre analyse, même si elle constitue une tentative après coup de rendre compte d'un geste incompréhensible, même s'il comporte plus d'une raison, mais plutôt le trouble qui l'entoure, les signes qui émergent de cette rationalisation qui échoue, signes qui nous indiquent à quel point Pierre Rivière était engagé depuis l'enfance dans un trouble que les psychiatres d'alors appelaient monomanie homicide et que nous percevons aujourd'hui comme un ratage du processus d'adolescence, voire comme une psychose pubertaire dont l'issue suicidaire et mélancoliforme apparaitra plus tard au cours de son incarcération. Le texte du mémoire, au même titre qu'une parole échangée dans une relation à un autre (parole initialement adressée au juge avant de devenir texte) nous donne des nouvelles du monde psychique de Pierre à son insu. C'est cet aspect que nous retiendrons pour mettre en évidence l'effort démesuré que fait Pierre pour se penser, pour rendre intelligible un acte qui continue à lui échapper malgré toutes les explications qu'il donne au juge et dont le Mémoire porte la trace.

C'est à la suite d'un certain nombre d'événements (conflits violents et procédures judiciaires entre les parents, sentiments de persécution de la mère, pression de plus en plus forte exercée par la grand-mère paternelle) et de non événements (la puberté n'entraine pas une entrée en adolescence chez Pierre, l'infantile ne laisse pas place au pubertaire) que Pierre Rivière en vient à concevoir ses crimes. C'est l'histoire de ce cheminement qu'il relate dans son Mémoire, avec parfois beaucoup de détails, toujours avec sincérité. Du point de vue narratif, le Mémoire se donne comme un récit factuel au plus près d'une réalité observable, sans être pour autant dénué d'émotion, d'une certaine capacité autoréflexive. Le récit se veut objectif, explicatif, avec un souci évident de faire comprendreà son lecteur les raisons de son acte. Pour autant, ce récit traduit plutôt une tentative désespérée (de se faire comprendre) plutôt qu'une façon de se construire un passé (Aulagnier, 1989). Dans ses descriptions, il se présente comme ayant « des singularités » qui le distinguent des autres, comme sa pratique du dénichage, vestige de l'enfance qui, chez lui, ne disparait pas avec l'adolescence. Comme si Pierre Rivière restait en deçà de la génitalisation (il n'abandonne pas le langage des oiseaux pour le langage amoureux), en se maintenant à la fois dans la position d'enfant qui a peur des femmes (il criait « Le diable ! » chaque fois qu'il en rencontrait une) et à la fois dans une position toute puissante d'auto-engendrement qui le pousse à se sacrifier (s'auto-désengendrer) pour sauver son père.

Pierre Rivière pense sa vie comme une vie de sacrifice. Il se dit qu'il faut qu'il fasse tout pour sauver son père de la persécution dont il est victime de la part de sa mère. C'est son code d'interprétation des relations parentales. Il va tout mettre en œuvre pour réaliser ce projet, convaincu que ce meurtre est la seule issue, puisque c'est elle, sa mère, qui organise la dépression de son père. Il voyait son père se déprimer, menacer de se suicider, revenir du puits en disant qu'il allait s'yjeter, etc. Très touché par ces scènes, Pierre Rivière est encouragé par le clan paternel, la grandmère paternelle surtout, pour rester solidaire de son père contre sa mère. Au gré des ouvrages qu'il dévore tout au long de son adolescence, des lectures qu'il effectue sans partage, dans un rapport autodidacte à l'écriture (il lit les ouvrages que colportent les vendeurs de village en village, souvent des livres pieux dont il s'inspire, des manuels d'Histoire de France aussi, qu'il interprète d'une façon paranoïaque) il va se convaincre dans une identification héroïque que, comme Jésus s'est sacrifié pour sauver les hommes, comme tel ou tel héros biblique qui se sacrifie pour sauver les siens, lui aussi va se sacrifier, sacrifier sa jeunesse, son avenir, sa vie, pour sauver son père de la dépression.

Le monde interne de P. Rivière se divise ainsi en deux parties injoignables, l'une bonne mais persécutée (à l'image de ce que vit chacun des parents), l'autre mauvaise et persécutrice. L'explication du crime est une tentative de réduire ce clivage. Mais P. Rivière n'y parvient pas vraiment et sa mort est déjà inscrite dans cet acte mélancoliforme du crime familial.

Plutôt que de s'opposer au père pour rivaliser avec lui, le tuer fantasmatiquement et prendre sa 
place auprès de la femme du père, comme cela se produit dans les œdipes adolescents ordinaires, $\mathrm{P}$. Rivière tue sa mère réellement, il renonce se faisant à cet œdipe pubertaire - qui aurait pu le pousser à rencontrer des femmes - pour une relation sadique d'allure incestueuse réalisée dans le meurtre sanguinaire où le corps à corps avec la mère peut enfin avoir lieu. On pourrait dire que ce matricide constitue un double meurtre, celui de la mère en tant que femme et celui du père dont il prendrait réellement la place auprès de la mère. C'est en ce sens-là que l'on peut parler d'un ratage dans le processus d'adolescence, au sens où Pierre échoue à symboliser, à conflictualiser dans l'œdipe l'investissement des objets parentaux.

Le récit de l'histoire familiale que donne $\mathrm{P}$. Rivière dans son mémoire laisse transparaittre le sentiment d'abandon dans lequel l'aurait laissé sa mère dès sa naissance et surtout dès la naissance de sa sœur cadette, Victoire. Se sentant privé de l'amour maternel, privé du rapport au corps de la mère que $\mathrm{P}$. Rivière décrit comme meurtri par la première grossesse, il envie sa sœur Victoire, elle qui est dans la confidence de sa mère, comme l'enfant aimée, un double narcissique de l'image maternelle. C'est donc elle qu'il tuera en même temps que la mère. Plus complexe est le meurtre du plus jeune frère, Jules, le préféré du père, cette fois. Pierre explique dans son mémoire qu'il l'a tué justement parce qu'il était le préféré du père et qu'ainsi, son père n'aurait pas à éprouver le sentiment d'une dette à son égard, dette relative au fait que le fils aurait délivré le père de la persécution de sa femme. L'abnégation va jusqu'à cette horreur et révèle s'il en était besoin la dimension narcissique de toute cette construction.

La sexualité de Pierre reste narcissique, en effet, et prégénitale; il ne comprend pas ce qui se passe dans la relation passionnelle entre ses parents. Il interprète sans recul, littéralement, leur rapport comme une lutte à mort en s'identifiant à la cause de son père. C'est cette identification à la victime qui le pousse à la vengeance. Pierre Rivière reste imaginairement collé à son père dans une position homosexuelle de refus de la complémentarité des sexes. Pas de scène primitive ni de scène pubertaire : à leur place, une imagination d'un coït sadique d'où le père sort châtré par la mère.

La scène du crime, ses motifs et sa réalisation sont étroitement liés au contexte politique de la Restauration qui suit la période violente de la Révolution française couronnée en quelque sorte par la décapitation du roi. Pierre Rivière, à l'image de son père, s'accroche au temps de l'Ancien Régime et prend appui sur les textes bibliques qu'il comprend à sa façon (autodidacte) sans référence aux commentaires de la tradition. De son côté, la mère vit dans son temps, celui de l'égalité citoyenne entre les hommes et les femmes et réclame justice contre son mari qui lui prend ses enfants. En effet, après chaque naissance, le père ramène l'enfant chez ses propres parents avec lesquels il vit, renforçant le sentiment de persécution maternel. Pierre Rivière s'appuie sur les textes bibliques comme dans ce passage où Judith coupe la tête d'Holopherne, ce général ennemi d'Israël. P. Rivière souffre de ce que les femmes aient tant de pouvoir sur les hommes et regrette le temps où le père avait droit de vie et de mort sur sa femme et sa descendance. En tuant sa mère (dans une scène inversée par rapport à Judith et Holopherne) - il la décapite, quasiment -, il espère rétablir cet ordre ancien et sauver ainsi son père de la furie vengeresse de sa mère. Cette vision paranoïaque de la relation entre les hommes et les femmes est aussi une tentative de revenir à un autre temps, celui de la toute-puissance infantile. Ce temps historique qui sert de toile de fond à l'histoire singulière de Pierre fait apparaître les correspondances qui s'établissent entre réalité historique et réalité psychique. P. Rivière cherche à éviter l'angoisse de castration ou plutôt à s'instaurer comme le castrateur suprême en se situant littéralement du côté du manche plutôt que de celui de la cognée. Faute de pouvoir symboliser ce rapport au manque qui lui donnerait la possibilité de désirer sexuellement l'autre, Pierre Rivière suture ce manque et rétablit le système binaire phallique castré qui l'empêche d'accéder à la rencontre avec l'autre dans la différence génitale et la complémentarité des sexes. 


\section{LE CAS DE PIERRE RIVIÈRE À LA LUMIÈRE DU PROCESSUS D'ADOLESGENCE : HISTOIRE D'UN RATAGE}

A la lecture de son Mémoire, on perçoit les troubles graves du processus de subjectivation que présente Pierre Rivière. Dans sa vie, il n'y a pas de place pour lui comme sujet. La famille apparaît comme scindée en deux (clan paternel d'un côté, clan maternel de l'autre, avec la haine comme lien) et chacune de ses parties semble fusionnée, les places de chacun ne sont pas distinguées au-delà de ce clivage, le climat est fait d'incestualité, chaque parent continuant à vivre avec les siens. Au lieu d'offrir à l'enfant une configuration œdipienne structurante, c'est le tableau de la haine - celle qui déchire et oppose les clans qui domine, avec son lot de projections et d'interprétations. Pierre Rivière prend tout à la lettre, ce qui donne une lecture délirante des faits, comme est délirante sa rencontre avec les textes qu'il lit sans référence au sens commun ni aux commentaires issus de la tradition et de la culture.

Dans ce contexte, le déni de l'altérité alimente la projection de la violence interne et le clivage pathologique entre bon et mauvais objet. Ce clivage se retrouve au niveau des familles paternelle et maternelle qui ne s'ignorent pas l'une l'autre, mais se déchirent en permanence, engageant Pierre dans cette vision séparée du couple parental. La haine qui lie père et mère dans la représentation que se fait Pierre de leur relation le pousse à prendre parti pour le plus faible (le père), induisant ainsi une identification héroïque au sauveur (le Christ), à une filiation narcissique (Guyotat, 1989) qui renverse l'ordre des générations et pousse le fils à venger le père.

Le motif du crime s'enracine profondément dans cette vision très personnelle de l'histoire familiale où domine la relation passionnelle et destructrice entre les parents. Cette impossibilité de trouver un espace familial vivable pour soi sert de contexte au crime qui peut être compris comme une difficulté personnelle de P. Rivière à se séparer de ses objets parentaux (à l'image de ses parents qui ne parvenaient pas à quitter les leurs), à élaborer sa vie pulsionnelle et la violence pubertaire qui se déchaîne dans le matricide et le double fratricide.

Le passage à l'acte révèle l'effondrement du monde interne de P. Rivière, l'étendue de sa construction délirante. Le commentaire qu'en fait $\mathrm{P}$. Rivière lui-même dans son Mémoire donne à entendre le fantasme central qui l'anime : sauver le père (se protéger de la destructivité d'une mère archaïque et prégénitale toute puissante) et se sacrifier (s'auto désengendrer dans un mouvement de toute puissance narcissique dans lequel l'auteur du crime est enfermé), pour éviter d'avoir à être confronté par personne paternelle interposée au risque de la séparation. Mieux vaut agir la séparation (tuer) plutôt que d'être quitté, abandonné et éventuellement châtré.

\section{DE LA GRÂCE ROYALE À LA GRÂCE PARENTALE}

A l'issue du procès, Pierre Rivière est condamné à mort, puis gracié par le Roi qui commue sa peine en prison à vie, bénéficiant ainsi de la reconnaissance de son état pathologique (folie homicide) au moment des faits. Dans l'espace familial, la grâce parentale pourrait être envisagée comme le soutien narcissique que les parents doivent à l'adolescent au moment où le gué est franchi à moitié et où l'adolescent est fragilisé sur le plan narcissique, violenté de l'intérieur par le pubertaire (Gutton, 1991) lui-même et de l'extérieur par les objets qu'il rencontre, les relations qu'il noue et qui deviennent souvent menaçantes pour lui. Les parents sont eux-mêmes soumis à un paradoxe : avoir à soutenir, et même à armer le bras qui va les tuer fantasmatiquement (Gutton, 1989), soutenir le devenir adolescent qui passe par l'attaque fantasmatique des images parentales et le meurtre symbolique des parents. Etre parent, accorder sa grâce en tant que parent à l'enfant devenant adolescent, c'est soutenir ce mouvement symboliquement meurtrier qui est la voie d'accès à la résolution du conflit pubertaire. Pour que l'adolescent puisse traverser son adolescence, il faut qu'il ait pu se confronter aux 
parents, dans un espace où il est contenu et non pas où il est agi. Cela demande aux parents de voir le conflit adolescent non pas comme violence destructrice, mais au contraire comme une force créatrice en germe. C'est précisément grâce au soutien narcissique parental que cette destructivité se transforme pour se mettre au service de la culture. C'est souvent lorsque les parents contre-réagissent à la destructivité de l'adolescent en ayant eux-mêmes recours à la violence que le risque du passage à l'acte à l'adolescence est le plus élevé.

Les adultes, et les parents de Pierre Rivière tout particulièrement, apparaissent en grande difficulté pour venir en aide à cet adolescent en danger. Le soutien narcissique parental ne fonctionne pas, chacun des parents étant engagé dans un rapport de force avec l'autre. Les parents ne protègent pas leur enfant devenant adolescent de la violence des excitations qui se manifestent à de nombreuses occasions (conduites singulières avec de jeunes enfants, agressivité mimétique envers sa sœur Victoire, puisque Pierre Rivière battait sa sœur comme le père le faisait avec sa femme, violence et cruauté contre les animaux, peur des femmes, peur de commettre l'inceste). Pierre Rivière semble particulièrement exposé à cette violence conjugale qui illustre sur la scène de la réalité externe le conflit violent qui a lieu dans son monde interne. La violence destructrice qui oppose les parents est à l'image de la violence interne à laquelle est confronté Pierre. La violence du fantasme concernant la puissance phallique de la mère attaque ses capacités d'élaboration et de névrotisation de cette conflictualisation interne.

\section{LE PARRICIDE N'EST PAS LA RÉALISATION D'UN FANTASME CEDIPIEN, MAIS LE MEURTRE DE CE FANTASME}

Le parricide peut faire penser à un accomplissement du fantasme incestueux : tuer le père pour avoir la mère. Mais, en réalité, le parricide surinvestit le père dans un lien homosexuel. Il n'a pas désinvesti cette voie pour la conquête de l'ob- jet incestueux maternel. C'est dans ce sens-là que l'accomplissement du parricide est une impasse dans le processus pubertaire. Il s'agit dans ces cas de parricide d'un investissement pervers de la violence érotisée dans le lien sadomasochiste qu'entretient l'adolescent avec le parent homosexuel. Il conviendrait ici de développer également cette approche de l'investissement pervers de l'objet parental et de l'érotisation de la violence, dans la perspective de mettre en évidence le rôle majeur que joue sur le plan pulsionnel la destructivité comme conséquence de la désintrication pulsionnelle, la haine ayant pris la place de l'amour dans le lien à l'objet. On observe également dans ces cas de parricide que les instances psychiques ne semblent pas s'être différenciées et que les espaces psychiques des membres de la famille sont confondus. Nous aurons l'occasion d'y revenir un peu plus loin. L'investissement pubertaire est sacrifié au profit d'une fixation narcissique homosexuelle, traduisant l'impossibilité pour l'adolescent d'aborder les fantasmes incestueux et parricides qui constituent ces organisateurs psychiques dont nous avons mentionné l'importance dans l'évolution du processus d'adolescence.

\section{LE MATRICIDE EST-IL UN PARRICIDE AU FÉMININ?}

Si le parricide n'est pas la réalisation du fantasme œdipien, le matricide serait-il un parricide au féminin ? Le matricide est-il l'équivalent du parricide pour la fille ? S'agit-il du meurtre du parent œdipien rival ? D. Brun (1990) propose de considérer que le matricide « procède de la recherche de la femme dans la mère ». Cette perspective envisage le matricide comme un fantasme de la série oedipienne, qui aurait pour fonction de permettre à la fille, en tuant la mère, d'accéder aux identifications féminines. Tuer la mère en la femme pour que puissent s'arrimer des identifications de femme à femme en quelque sorte. Cette position ouvre de réelles perspectives quant à une théorie de la sexualité féminine. Ce fantasme est porteur d'une identification au 
même, un peu sur le mode du fantasme parricide qui permet au garçon de se situer par rapport au rival, à l'autre semblable. En réalité, comme nous venons de le voir, le parricide comme meurtre du père n'est pas la réalisation du fantasme de meurtre, mais serait ce que j'ai proposé d'appeler un meurtre du fantasme (Marty, 1989). Par ailleurs, il y a un changement de registre entre le parricide et le matricide. Si l'un comme l'autre constituent des impasses quant au processus pubertaire, quoiqu'il faille examiner chaque cas précisément, il apparaît que le matricide constitue un risque majeur d'évolution psychotique. On ne saurait tuer la mère, car tuer la mère c'est tuer l'objet primordial, c'est attaquer la matrice même de la vie (matricide), l'origine de soi, c'est scier la branche sur laquelle on est assis. Le matricide ne constitue pas un équivalent féminin du parricide, il éclaire l'acte criminel dans ce qu'il comporte de plus primitif quant au fonctionnement psychique. La mère tuée par la fille ne représente qu'une part infime des meurtres familiaux, le parricide au sens large étant davantage le fait des garçons que des filles ( 1 fille pour 9 garçons). Les crimes parricides et matricides connus dans l'antiquité et la mythologie sont d'ailleurs presque tous commis par des hommes. Oreste en est le héros le plus célèbre. Un fils tue sa mère pour venger son père. Dans «Agamemnon», on assiste au retour du chef victorieux de Troie, Agamemnon, roi des Rois. Déjà, Clytemnestre, son épouse, projette de le tuer, lui et Cassandre sa maîtresse, pour venger sa fille Iphigénie qu'Agamemnon a sacrifiée aux dieux afin de s'assurer leur faveur. L'amant de Clytemnestre, Égisthe, l'aide dans le double meurtre pour venger son propre père Thyeste, banni par le père d'Agamemnon, Atrée. Mais Égisthe est un lâche et il laisse Clytemnestre se charger de la besogne.

Dans les «Choéphores», il s'agit encore de la vengeance d'un meurtre par un autre meurtre. Oreste tue sa mère et l'amant de celle-ci, Égisthe. Une fois le crime accompli, il est poursuivi par les Érinyes qui demandent vengeance.

Enfin, dans «les Euménides» a lieu le procès d'Oreste, son acquittement et l'instauration d'une loi qui allie la vigueur ancienne des Furies vengeresses et la pondération d'Athéna. Celle-ci réunit un aréopage de sages pour rendre une justice humaine en accord avec les dieux (devenus Euménides), qui veillent sur le respect des lois.

L'Orestie est le récit d'une transformation. A la justice vengeresse du crime qui appelle le crime, du «œil pour œil, dent pour dent», répond une justice plus humaine, mais plus solide qu'un rempart parce qu'elle s'élève au-dessus des intérêts partisans, et allie les lois du sang et le devoir de vengeance aux lois de la Cité et au devoir de solidarité entre les humains.

Comme Hamlet ou Rodrigue, Oreste doit venger son père dans un code d'honneur qui lui en fait devoir. Comme Oreste, Pierre Rivière est un fils qui tue sa mère pour venger l'honneur de son père. Tous deux désignent les dieux comme commanditaires du crime. Les Érinyes familiales, déesses de la vengeance, poursuivent les meurtriers et réclament à leur tour vengeance. C'est le cercle sans fin du meurtre pourvenger le meurtre.

Dans le procès qui les juge, le tribunal, l'Aréopage dans l'Orestie, a bien du mal à décider de la culpabilité des accusés et il faut l'intervention souveraine d'Athéna pour Oreste, et du Roi pour Pierre Rivière, pour qu'une issue soit trouvée : la grâce. Le matricide et le procès d'Oreste ont été l'occasion d'établir cette justice, faite d'équité et d'humanité qui dispense l'individu du devoir de vengeance personnelle. (On notera, en outre, dansl'Antigone de Sophocle, qu'Eurydice, femme de Créon, lui-même père d'Antigone, se donne la mort, tout comme la mère d'Hamlet). On voit que dans tous ces cas, il s'agit d'un meurtre matricide qui s'inscrit dans un code de l'honneur, dans un système de vengeance. Ici, la violence se met au service d'un règlement de compte, d'une vendetta. Nous devons transposer ce système de valeur propre aux sociétés dans lesquelles un membre de la famille est chargé par les autres de faire régner la justice par la vengeance de l'honneur perdu. C'est une violence qui s'exerce en lien avec le culte des morts, c'est une violence ritualisée, socialisée dans laquelle l'individu n'est qu'un instrument d'un ordre social qui se maintient grâce au peu de cas qui est fait de l'individu. Il nous faut transposer ce système de régulation 
des tensions sociales de l'échelle du groupe à celle de l'individu, de celle des sociétés traditionnelles aux sociétés modernes, pour examiner la nature de cette violence du matricide commis par les adolescents, à la lumière d'une théorie du pubertaire. Le cas de Pierre Rivière est intéressant aussi au titre de cas charnière dans l'histoire des mentalités, c'est le sens du choix de ce cas par Michel Foucault, dans la mesure où il traduit une évolution qui se déroule depuis le début du XIXème siècle. À la violence rituelle d'une société qui privilégie l'appartenance au groupe, sous le règne d'un chef, à la valorisation de l'individu, qui caractérise notre société moderne, apparaît la violence qui ne se contient plus, qui déborde l'ordre policé du social. Ce débordement est pour Foucault le signe de la naissance de la psychiatrie, de l'enfermement dans un système de pensée, où la norme contraint l'individu à se plier à l'ordre et où le dépassement des limites ainsi tracées sera sanctionné par la police mentale que représente à ses yeux la psychiatrie. À la violence de la vengeance succède celle de l'ordre, Pierre Rivière devenant à cette occasion un modèle permettant de penser cette transformation du regard du social sur ses limites, ses tolérances, sa capacité à intérioriser la violence.

Pour nous, Pierre Rivière est surtout l'exemple d'une errance adolescente au moment où se met en place la quête de l'objet adéquat, c'est un exemple pathétique d'un ratage du processus pubertaire, le crime matricide traduisant la difficulté de Rivière à élaborer sa violence interne pour la mettre au service d'un processus de subjectivation. Le crime de Rivière traduit à nos yeux la difficulté à constituer un objet maternel.

Lorsque le garçon accomplit un matricide, il tue un parent phallique, castrateur du père. Lorsqu'il tue la mère, le garçon cherche à faire exister le père. Il est à la recherche d'un étayage narcissique, homosexuel, suffisamment structurant pour pouvoir ensuite, mais ensuite seulement, attaquer le père dans l'affrontement œedipien et dans la conquête de la mère. C'est dans ce sens-là que j'ai analysé le cas de Pierre Rivière.

Lafille qui tue la mère ne tue pas sa rivale œdipienne, mais une figure archaïque d'elle-même.
C'est à un meurtre de sa capacité à se représenter qu'elle procède, ayant recours au meurtre comme mode de résolution d'une problématique de collage à l'objet primitif qui lui interdit toute élaboration du lien à l'objet. Le matricide pour la fille n'est pas une attaque de la mère œdipienne rivale, mais la tentative d'anéantissement d'un lien pathologique à l'autre sur le mode de l'indifférenciation ou du miroir. Le matricide semble en définitive être un meurtre de la représentation de soi, une attaque de sa propre image au miroir, ce double spéculaire qui constitue la trame identifiante pour l'enfant dans son rapport à son propre corps pris comme réalité distincte du corps maternel, et, par là, comme espace psychique différencié de l'objet primordial. Le meurtre de la mère n'est donc pas celui de l'objet narcissique, étayant la subjectivité naissante de l'enfant, mais la destruction d'un double collant à l'image de soi comme la partie placentaire du fotus destinée à rester attachée au corps maternel, détachée de l'enfant. Le matricide serait de ce point de vue, le meurtre de la part mortifère de la matrice, la mère étant identifiée à ce reste de la vie à son origine, à ce jumeau mort-né, destiné à l'oubli, à un état d'inexistence. L'adolescent matricide identifie sa mère à cette partie du ventre originel pour naître à la vie sans reste, pour s'acquitter de toute dépendance, de toute dette à l'égard de la matrice. C'est comme s'il cherchait à faire disparaître toute trace de son lien à l'ombilic, dont le nombril est le témoignage, pour finalement s'auto-engendrer. L'adolescent matricide cherche à se rendre vivant, à partir de cette expérience de la séparation primitive d'avec la partie de lui destinée à la mort et à l'oubli qu'est ce double, ce jumeau fantasmatique que représente le placenta.

Mais pourquoi s'en prendre à la mère ? Le matricide est en quête d'une reconnaissance impossible, d'un regard fondateur qui lui donnerait la certitude qu'il vaut plus que son reste. Habituellement, l'enfant acquiert cette certitude qui lui offre la voie d'un narcissisme constitutif de la subjectivité par le regard maternel qui le réconcilie avec le double au miroir, pour finalement lui donner la priorité sur son reflet. La mère préfère l'enfant à son image. C'est dans ce sens que j'en- 
tends S. Leclaire parler de « On tue un enfant » (1975). Il faut tuer l'enfant fantasmatique pour que puisse advenir l'enfant réel. Dans la problématique de l'adolescent matricide, on peut se demander si cette réconciliation de l'enfant avec son double fantasmatique a eu lieu. Le matricide est la mort donnée à cette infertilité du lien mère enfant, lien qui a échoué à fonder l'assise narcissique de l'enfant. Il ne s'agirait pas d'en finir avec la mère, mais plutôt de souffrir de n'avoir jamais pu commencer avec elle.

Le matricide traduit dans une réalité sanglante la réalité interne de l'adolescent meurtrier qui souffre d'avoir en lui une mère morte au moment où il devrait (re)trouver l'objet maternel incestueux pour entreprendre sa quête d'un objet plus adéquat. L'adolescence, et plus précisément l'œdipe pubertaire confronte l'adolescent porteur d'une mère morte à ce non-avènement d'un lien à l'objet narcissique, à cette absence en lui d'un sentiment d'avoir été préféré à l'autre part de lui-même restée dans le ventre maternel, l'autre idéalisé par la mère, l'autre porteur des promesses que l'enfant fantasmatique faisait miroiter à la mère elle-même. Le double gémellaire, placentaire ou idéal, n'a pu être tué pour offrir à l'enfant né le sentiment qu'il valait mieux que cela.

Mais le matricide trouve encore un équivalent dans l'inceste mère-fils (Bunker, 1944). C'est un point de vue que j'ai également développé à la suite de C. Stein (1977) à propos de l'errance d'œdipe (Marty, 1994). Le parricide accompli par le héros est suivi d'un matricide, dans la mesure où posséder la mère c'est la tuer en tant que représentation de la mère. Pour J. Bergeret (1994), Edipe met à mort sa mère deux fois. D'abord comme mère phallique avec la sphinge, et une seconde fois avec la mère génitale dans la scène ultime dans la chambre royale, dont la conclusion par le suicide masque à peine le sens que revêt l'inceste consommé et son impossible consommation, devrait-on dire. La mère, c'est précisément celle que l'enfant ne peut pas posséder. C'est cette condition basée sur l'interdit qui fonde le repérage symbolique des places que chacun occupe dans l'espace familial et qui déter- mine les liens de parenté et de filiation. Or c'est ce lien symbolique qui est attaqué dans le matricide, le meurtre de la mère mettant en péril le repérage pré œdipien de soi. La signification des places qu'occupent les membres d'une famille tient essentiellement au fait que certaines sont interdites, et que toutes sont marquées. Il se trouve que le premier objet d'étayage des besoins et des satisfactions de ces besoins de l'enfant est le même objet que l'objet investi secondairement sur le plan libidinal. La mère est d'abord dans sa fonction première l'objet de satisfaction des besoins avant d'être objet de désir. Mais il se trouve aussi que la mère, première ou deuxième version, est l'objet du désir d'un autre. C'est cette configuration originale qui fait de l'objet maternel un objet investi culturellement, car il porte la marque de la présence d'un autre et de son désir. Cette marque atteste de sa place dans le désir de la mère et signifie à l'enfant que cet objet est déjà investi, déjà marqué. L'enfant est donc confronté à un ensemble de signes qui lui sont transmis comme autant de modes d'inscriptions dans le registre symbolique. L'enfant désire l'objet qui précisément est déjà l'objet du désir d'un autre. Cette tragédie offre à l'enfant les moyens de se repérer par rapport à cet autre, que ce soit dans la haine ou l'amour, l'ambivalence, la violence, le renoncement à l'hétérosexualité. L'interdit de l'inceste donne à l'enfant les marques symboliques de ce repérage, en lui indiquant la place du père, et par là celle de la filiation, donc la sienne. Commettre l'inceste, c'est subvertir ces places, c'est occuper une position qui n'est pas la sienne. L'inceste fait changer la mère de place en tant que personne, mais la fait mourir en tant que signe distinctif de repérage. L'inceste est le meurtre de la représentation de l'objet maternel, c'est une autre forme de matricide. L'inceste commis par les parents sur la personne de l'enfant est un équivalent de parricide (pris ici au sens large de meurtre parental), dans la mesure où le parent incestueux quitte la place de parent qu'il occupe au moment où il commet l'inceste. Il se met lui-même à mort comme parent.

Dans les différentes études portant sur les parricides commis à l'adolescence, il est remar- 
quable que beaucoup d'entre eux se soient produits dans des contextes familiaux de violences incestueuses. Dans les études récentes (Goudal et al., 1998) menées sur des crimes commis par des adolescentes, nous avons pu établir que la plupart d'entre elles avaient subi des violences sexuelles, récentes ou anciennes, et que leur procès avait été l'occasion pour elles de parler de ces violences subies. S'il n'est pas possible d'inférer la violence parricide uniquement de la violence subie par ces adolescents criminels dans une causalité trop linéaire et déterministe, il faut bien noter la fréquence de ces violences, qui pour ne pas avoir été toutes manifestes ont toutes été subies. Il convient maintenant de rechercher dans notre clinique de l'adolescence du côté des traumatismes précoces quelques lumières sur cette faculté singulière qu'ont certains adolescents à avoir recours à l'acte (parricide ou plus largement criminel) pour tenter de résoudre des énigmes existentielles source d'une souffrance indicible et pourtant agissante.

L'adolescence est source de tous les dangers comme elle est source de tous les possibles, de toutes les créativités. Mais, le « remake » du scénario œdipien infantile, pour reprendre l'expression d'A. Birraux (1990), potentialise les risques de passage à l'acte, les risques d'un recours à des agir qui trouvent leur origine dans la puissance, voire la violence des fantasmes pubertaires. Le terme anglais de remake, repris dans notre langue comme un néologisme qui signifie reprise modifiée, transformée d'une œuvre originale, traduit assez bien ce travail mutatif de la puberté qui prend sa source dans le vécu infantile œdipien. Le matricide, comme le parricide, expriment le mode économique de gestion des conflits qui ne parviennent pas à s'intérioriser. Ils traduisent la difficulté à se séparer des objets parentaux, et ce, depuis l'enfance, provoquant un collage traumatique au moment crucial de la puberté, moment où précisément se rejouent à la fois le lien œdipien aux objets parentaux et à la fois la nécessité d'intérioriser ce lien, pour se détacher des parents. L'adolescent parricide tue un objet qui a échoué dans sa fonction d'étayage. L'objet parental étaye narcissiquement l'enfant lorsqu'il lui offre la possibilité du lien et de la séparation, de l'illusion dans la création et la trouvaille de l'objet et de la désillusion qui marque la fin de la toute-puissance infantile. Dans le cas des adolescents parricides, cette fonction d'étayage a échoué, les parents et les enfants restant imaginairement collés, cette adéquation quasi fusionnelle explosant au moment de la puberté. La proximité des parents dans l'espace psychique de l'adolescent, l'espace psychique n'étant pas là un espace de pensée, mais un espace de confusion, ne permet pas de dégager la place de l'objet adéquat. Il n'y a pas de jeu possible entre les places, elles sont soudées dans une communauté imaginaire, qui dans le cas de matricide évoque la psychose.

Résumons : dans le matricide, ce n'est pas la mère œdipienne qui est tuée. A l'adolescence, le matricide apparait comme un ratage dans la quête de l'objet adéquat, résultant d'un collage à l'objet maternel primaire. Le meurtre de l'objet maternel traduit la difficulté originelle de la séparation entre l'enfant et la mère. Ce meurtre révèle la puissance de la destructivité qui est à l'œuvre dans des relations aliénantes, comme si cette folie du lien ne pouvait que se trancher et non se dénouer, comme si l'acte était seul en mesure de venir à bout de cette pathologie du lien.

\section{CONCLUSION}

Le passage à l'acte meurtrier (matricide et double fratricide) de Pierre Rivière apparaît comme un crime altruiste d'allure mélancolique. La perte de l'amour maternel est insurmontable, la haine qui conduit au crime se retourne contre l'auteur de l'acte. Ce passage à l'acte meurtrier traduit l'impasse narcissique dans laquelle se trouvait Pierre Rivière depuis l'enfance, impasse narcissique qui ne pouvait qu'entraver le processus d'adolescence et le mettre finalement en échec : au lieu de se séparer des objets familiaux pour aller à la conquête d'objets étrangers, non incestueux, Pierre Rivière vient se coller imaginairement aux scènes sexuelles de ses parents, entrant dans ces scénarios intimes où il entend 
jouer les premiers rôles. La lecture du Mémoire montre à quel point la logique qui y est déployée pour rendre compte du crime se révèle être davantage para logique que rationnelle et qu'elle se heurte à une autre logique inconsciente, celle-là, où c'est la sexualité infantile qui guide les gestes du criminel. Mais dans ce cas, le sexuel infantile n'est pas refoulé par le pubertaire, il envahit la scène au contraire sur un mode de fonctionnement où règnent les processus primaires et où l'accès au registre de la symbolisation des liens aux objets parentaux est empêché par les clivages, les dénis qui saturent l'espace psychique familial. Le raisonnement de Pierre Rivière et les arguments qu'il avance pour tenter de justifier ses crimes soulignent l'organisation narcissique de sa sexualité, loin de l'investissement génital du corps et de la psyché tel qu'il se produit habituellement au moment de l'adolescence.

Dans cette histoire familiale, les places sont confondues, l'enfant Pierre Rivière ne semble pas avoir eu d'espace pour se penser en dehors des conflits parentaux. Il est entièrement envahi par cette problématique conjugale et familiale de la haine et du clivage, emporté par cette vision du monde où il ne trouve d'autre issue que d'agir pour éviter d'être agi. Le sacrifice qu'il fait de sa vie pour sauver son père illustre le fait que sa solution ne peut être que grandiose, dans un registre narcissique enflammé et en même temps réduit à rien par sa mort programmée. Le sens que cherche Pierre pour sa propre vie a ainsi été recouvert par la problématique familiale violente et envahissante qu'il mettra en acte et en scène, faute de pouvoir y tenir un propre rôle de sujet. Sa vie personnelle se confond alors avec la problématique familiale. Il devient acteur par le passage à l'acte pour devenir un Sauveur. Comme parfois, celui qui sauve disparaît, laissant la place à celui qu'il a sauvé. Mais là c'est dans un ordre inversé : le fils se sacrifie pour donner vie au père.

\section{BIBLIOGRAPHIE}

Aulagnier, P. (1989). Se construire un passé. Journal de la psychanalyse de l'enfant, 7, 191-220.

Bergeret, J. (1994). La violence et la vie. Payot.

Birraux , A. (1990). L'adolescent face à son corps. Éditions Universitaires.

Brun, D. (1990). La maternitéet le féminin. Denoël. Bunker, H. A. (1944). Mother-murder in myth and legend. A psychoanalytic note. Psychoanalytic Quaterly, 13, 198-207.

Goudal, M. C., Lelandais, F., Marty, F. \& Proux, E. (1998). Violences au féminin. Jeunes, femmes et violentes. Psychiatrie française, 4/97, 67-94.

Gutton, PH. (1989). Inimitable grandiosité du père. Adolescence, 7(2), 53-68.

Gutton, PH. (1991). Le pubertaire. PUF.

Guyotat, J. (1989). La filiation narcissique, In J. Guyotat (Ed.), Mort, naissance et filiation. Masson.

Kaës, R. (2009). Les alliances inconscientes. Dunod.

Leclaire, S. (1975). On tue un enfant. Editions Seuil.

Marty, F. (1989). Hercule ou la colère des Dieux. Adolescence, 7(1), 189-195.

Marty, F. (1994). Errer après son matricide. Adolescence, 23, 175-182.

Racamier, P.-C. (1980). Les schizophrènes. Paris, Payot.

Racamier, P.-C. 1989. Antoedipe et ses destins. Apsygée Éditions.

Rivière, P. (1835). Moi, Pierre Rivière, ayant égorgé ma mère, ma sœur et mon frère... Un cas de parricide au XIX ${ }^{e}$ siècle présenté par M. Foucault. Gallimard, 1973.

Stein, C. (1977). La mort d'eedipe. Gonthier Denoël. 


\title{
Matricídio : uma figura de laços familiares violentos
}

Resumo

Se os assassinatos familiares não são os atos mais frequentes no registo da violência intrafamiliar, não deixam de ser exemplos paradigmáticos de violência na adolescência, ilustrando tanto a violência do adolescente, como a violência na adolescência. Esses assassinatos não são a realização de fantasias edipianas, mas, antes pelo contrário, o assassinato da fantasia edipiana. Refletem ainda uma fixação narcísica, tanto no adolescente como na família. A partir do caso de um adolescente com vinte anos na altura em que cometeu os seus crimes, gostaria de discutir a ideia de que o ato assassino intrafamiliar é muitas vezes, e antes de tudo, a expressão de uma dificuldade de separação - física, mas sobretudo psíquica - de outros membros da família durante a adolescência. Essa dificuldade limita drasticamente a possibilidade de subjetivação. Esse crime de sangue é também o sinal da incapacidade de elaborar psiquicamente as transformações pubertárias, num contexto de incapacidade do adolescente em ajudar-se a si próprio. O papel do meio ambiente é predominante neste tipo de crimes, na medida em que o funcionamento psíquico da família se organiza muitas vezes sob o modo da indiferenciação e/ou da incestuosidade

Palavras-chave

Parricídio, Violência, Incesto, Fantasma de morte, Subjetivação

\section{Matricide, a figure of violent family ties}

\begin{abstract}
If family murders are not the most frequent acts in the register of violence in the family, and fortunately, they are nonetheless paradigmatic examples of violence in adolescence, illustrating the violence as well. of the adolescent than that of the adolescent. These murders are not the fulfillment of fantasies, but rather the murder of an Oedipal fantasy. They reflect a narcissistic functioning, that of the adolescent but also that of his family. Starting from the case of a teenager in his twenties when he committed his crimes, I would like to discuss the idea according to which the murderous intra-family act is very often, and first and foremost, the expression of a difficulty in separating - physically, but above all psychologically - from other members of the family during adolescence. This difficulty restricts the possibility of subjectifying oneself. This blood crime is also a sign of an inability to psychically work out pubertal transformations, against a background of inability to come to the aid of oneself. The role of the environment is prevalent in this type of crime, to the extent that the psychic functioning of the family is very often organized under the mode either of undifferentiation, or of incestuality.
\end{abstract}

